\title{
Cogan's syndrome with pyoderma gangrenosum: management of two uncommon disorders with aggressive presentation in a patient
}

\author{
Ezolene Pei-Chin Chua, ${ }^{1}$ Richard B Mallett, ${ }^{2}$ Sandeep Dahiya ${ }^{1}$
}

'Department of Rheumatology, Peterborough City Hospital, Peterborough, United Kingdom ${ }^{2}$ Department of Dermatology, Peterborough City Hospital, Peterborough, United Kingdom

\section{Correspondence to} Dr Ezolene Pei-Chin Chua, ezolene.chua@gmail.com

Accepted 1 May 2018

Check for updates

To cite: Chua EP-C, Mallett RB, Dahiya S. BMJ Case Rep Published Online First: [please include Day Month Year]. doi:10.1136/ bcr-2017-223876

\section{SUMMARY}

Pyoderma gangrenosum (PG) coexisting with Cogan's syndrome (CS) is uncommon, although cutaneous manifestations are known to develop in CS. A middleaged white female patient had chronic relapsing PG requiring ciclosporin and prednisolone. Despite receiving optimal doses of ciclosporin and prednisolone, she developed acute vestibulo-auditory symptoms as a result of CS. Ciclosporin was switched to methotrexate and prednisolone was increased. However, she continued to develop acute scleritis, requiring methylprednisolone pulses, and still had further flares of PG. Her methotrexate was held off when she developed severe pneumonia and she then received a trial of intravenous immunoglobulins (IVIG) for her recurrent leg ulcers. Unfortunately, she failed to respond to IVIG. Her ulcers eventually responded to six doses of monthly intravenous cyclophosphamide induction. Although CS is not an antineutrophil cytoplasmic antibodies (ANCA)-associated vasculitis, we used pulse cyclophosphamide, based on the experience of cyclophosphamide efficacy in severe ANCA-associated vasculitis (AAV). Following induction, both diseases currently remain under control with azathioprine as maintenance treatment.

\section{BACKGROUND}

Aggressive pyoderma gangrenosum (PG) developing in tandem with Cogan's syndrome (CS) has only been reported twice in the literature. We report a third case of aggressive CS and PG, successfully treated with intravenous cyclophosphamide induction and azathioprine as maintenance treatment. Guidelines on management are not available for both conditions due to their uncommon occurrence and lack of quality studies. CS has been recognised as a vasculitic phenomenon, but it is not usually associated with antineutrophil cytoplasmic antibodies (ANCA). While there are recommendations for treatment of severe ANCA-associated vasculitis (AAV), there is none for CS. Our patient had concurrent aggressive manifestation of both PG and CS; we treated her with intravenous pulsed cyclophosphamide and azathioprine, having recognised the efficacy of cyclophosphamide in AAV. The use of cyclophosphamide in AAV is one of the recommendations by the British Society of Rheumatology/British Health Professionals in Rheumatology (BSR/BHPR). We recognised that neither CS nor PG is an ANCA-associated vasculitis, but we considered the efficacy of intravenous pulsed cyclophosphamide in AAV and used it on our patient, due to a lack of controlled studies in the management of CS, which is a variable vasculitis.

\section{CASE PRESENTATION}

A 49-year-old Caucasian woman with diabetes mellitus (DM) and chronic obstructive pulmonary disease had recurrent ulcerations on both legs since 2014. The ulcers were thought to be due to DM and chronic venous insufficiency in view of their anatomical distribution. Her skin biopsy showed full thickness inflammation of the dermis and a small amount of fat necrosis in the superficial subcutis. Lupus band and fungal staining were negative, and there were no features of necrobiosis lipoidica and vasculitis. Arterial and venous Doppler scans of the legs did not detect peripheral arterial stenosis or deep and superficial venous insufficiency. Meticulous wound care supported by appropriate antibiotics helped with ulcer healing initially, but ulcerations over the legs continued on an undulating course over the next 2 years.

In 2015, multiple ulcerations over the shins reappeared spontaneously. Checks for antinuclear antibody (ANA), ANCA, rheumatoid factor, anticyclic citrullinated peptide and double-stranded DNA (dsDNA) were negative. A repeat skin biopsy showed neutrophilic aggregates within the dermis. Again, no vasculitic features were seen. Special stains excluded chronic atypical infections by fungus, mycobacteria and spirochetes. With the supportive findings of sterile neutrophilic aggregates and the clinical presentation of recurrent spontaneous ulcerations over the shin, the diagnosis of PG was concluded. She received oral lymecycline and topical Fucibet cream, followed by oral prednisolone up to $20 \mathrm{mg}$ daily, but ulcerations remained refractory. Oral ciclosporin $100 \mathrm{mg}$ twice a day was then added.

While receiving ciclosporin, the patient presented with meningism-like symptoms of headache, photophobia, visual blurring, neck stiffness, tinnitus, severe bilateral hearing loss, diplopia and fever. Bilateral gaze-evoked nystagmus was noted with right sensorineural hearing loss, suggesting acute dysfunction of the vestibulocochlear nerve. Other cranial nerves were normal.

Ophthalmology examination did not detect anterior or posterior ocular abnormalities. Review of her medications did not show any drugs that may cause her symptoms. Investigation for meningitis 
showed low cerebrospinal fluid (CSF) white cell count and low protein, with negative cultures. Opening pressure, during lumbar puncture, was not elevated, which excludes benign intracranial hypertension due to ciclosporin. Contrasted MRI of the brain was normal. Despite negative imaging and CSF investigations, she continued to develop distressing vertigo and profound sensorineural hearing loss. Her blood tests were significant only for chronic normochromic normocytic anaemia with haemoglobin of $75 \mathrm{~g} / \mathrm{L}$ and chronically elevated C-reactive protein of $283 \mathrm{mg} / \mathrm{L}$. Her renal, liver function, and repeated ANA, dsDNA and complements were normal.

Based on the acute presentation with audiovestibular dysfunction in a Meniere's-like syndrome and ophthalmological symptoms, she was diagnosed with atypical CS. We switched oral ciclosporin to oral methotrexate $20 \mathrm{mg}$ weekly and increased the dose of prednisolone.

One month into treatment with methotrexate and prednisolone, the patient developed a painful, red eye on the left. This time, ophthalmological examination found hyperaemia of her conjunctiva and sclera with scant cells in the anterior chamber. Posterior chamber examination was normal. She was started on intensive steroidal eye-drops and urgent intravenous methylprednisolone $500 \mathrm{mg}$ daily for 3 days for scleritis. Her treponema pallidum serology and Human Leucocyte Antigen (HLA) B27 were negative. Subsequent examination noted control of sclera inflammation and her prednisolone was increased to $60 \mathrm{mg}$ daily following the methylprednisolone pulses, while continuing methotrexate at $20 \mathrm{mg}$ weekly. Unfortunately following the flare of scleritis, the patient suffered from severe pneumonia requiring hospitalisation 2 months later and her immunosuppressants had to be held off briefly.

The patient developed further flares of PG despite resuming methotrexate and prednisolone shortly after her pneumonia. Despite intravenous methylprednisolone $1 \mathrm{~g}$ daily over 3 days, her leg ulcers (figures 1 and 2) were refractory to healing. An MRI of the leg did not detect complications of osteomyelitis or soft tissue collections. Intravenous immunoglobulin (IVIG) at $400 \mathrm{mg} / \mathrm{kg} /$ day for 5 days also failed to help with healing of PG ulcerations.

Considering the background diagnosis of a variable vasculitis, CS, superimposed by refractory PG, it was decided to treat the ulcers as for a vasculitic process. Since February 2017, she has received six doses of monthly intravenous cyclophosphamide at $15 \mathrm{mg} / \mathrm{kg}$. We used this treatment regimen based on previous experience of cyclophosphamide use in AAV, which is also

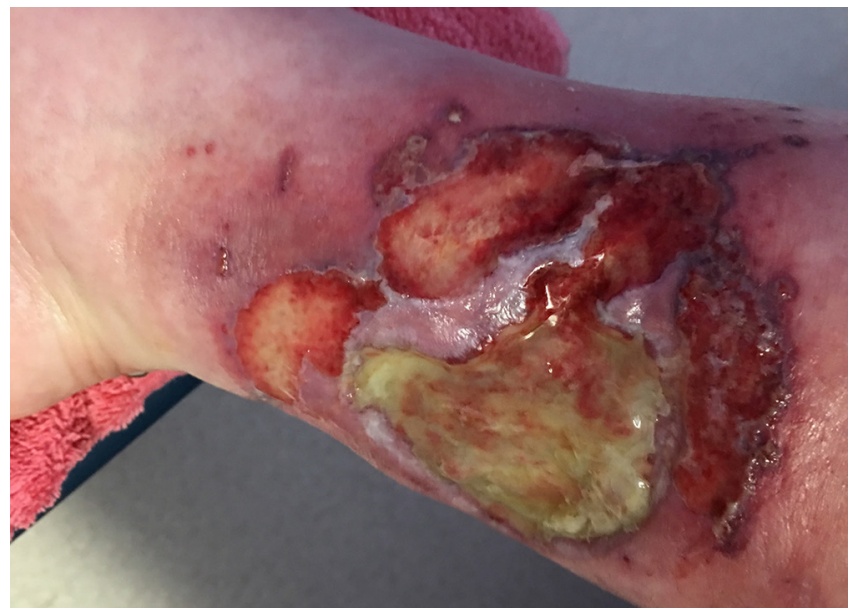

Figure 1 Right calf ulceration before cyclophosphamide induction.

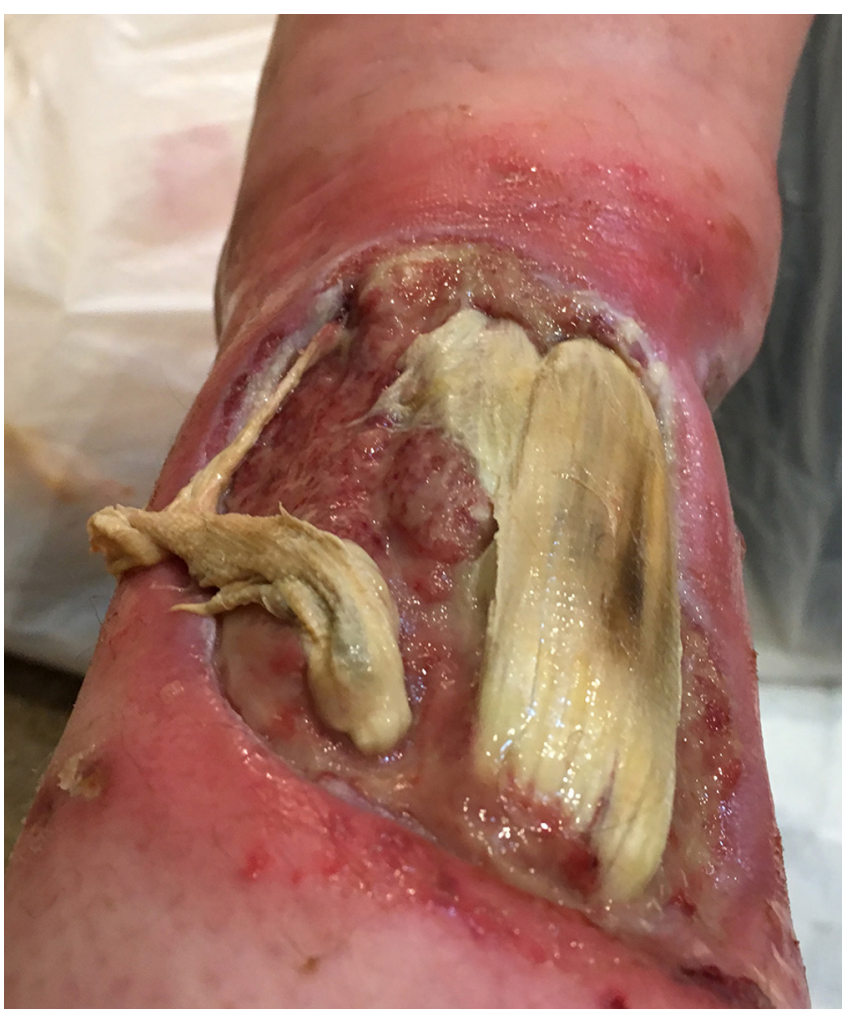

Figure 2 Left shin ulceration before cyclophosphamide induction.

described in the 2013 BSR and BHPR guidelines for ANCA-associated vasculitis. ${ }^{1}$ Her leg ulcers demonstrated good clinical response of wound healing (figures 3, 4, 5 and 6). Following induction with cyclophosphamide, azathioprine was used as maintenance treatment and she had no further flares of CS or PG.

\section{INVESTIGATIONS}

As summarised in 'Case Presentation'.

\section{DIFFERENTIAL DIAGNOSIS}

As discussed in 'Case Presentation'.

\section{TREATMENT}

As detailed in 'Case Presentation'.

\section{OUTCOME AND FOLLOW-UP}

Since February 2017, the patient has received induction treatment with six doses of monthly intravenous cyclophosphamide. This yielded good response, controlling further ulceration of PG, allowing the healthy granulation to develop at the base of the ulcers, shrinkage of most of the ulcers and epithelisation of previous ulcerations. Following this, her disease has remained suppressed with azathioprine as maintenance treatment.

\section{DISCUSSION \\ Cogan's syndrome}

CS is a rare autoimmune vasculitis with involvement of both the vestibulo-auditory and ophthalmic systems. Dr David Cogan first described the syndrome of non-syphilitic interstitial keratitis and acute vestibulo-auditory dysfunction in $1945 .^{23}$

Atypical CS was further proposed by Haynes et $\mathrm{al}^{4}$ in 1980, where other inflammatory eye lesions (such as scleritis, episcleritis, retinal artery occlusion, choroiditis, retinal haemorrhage, 


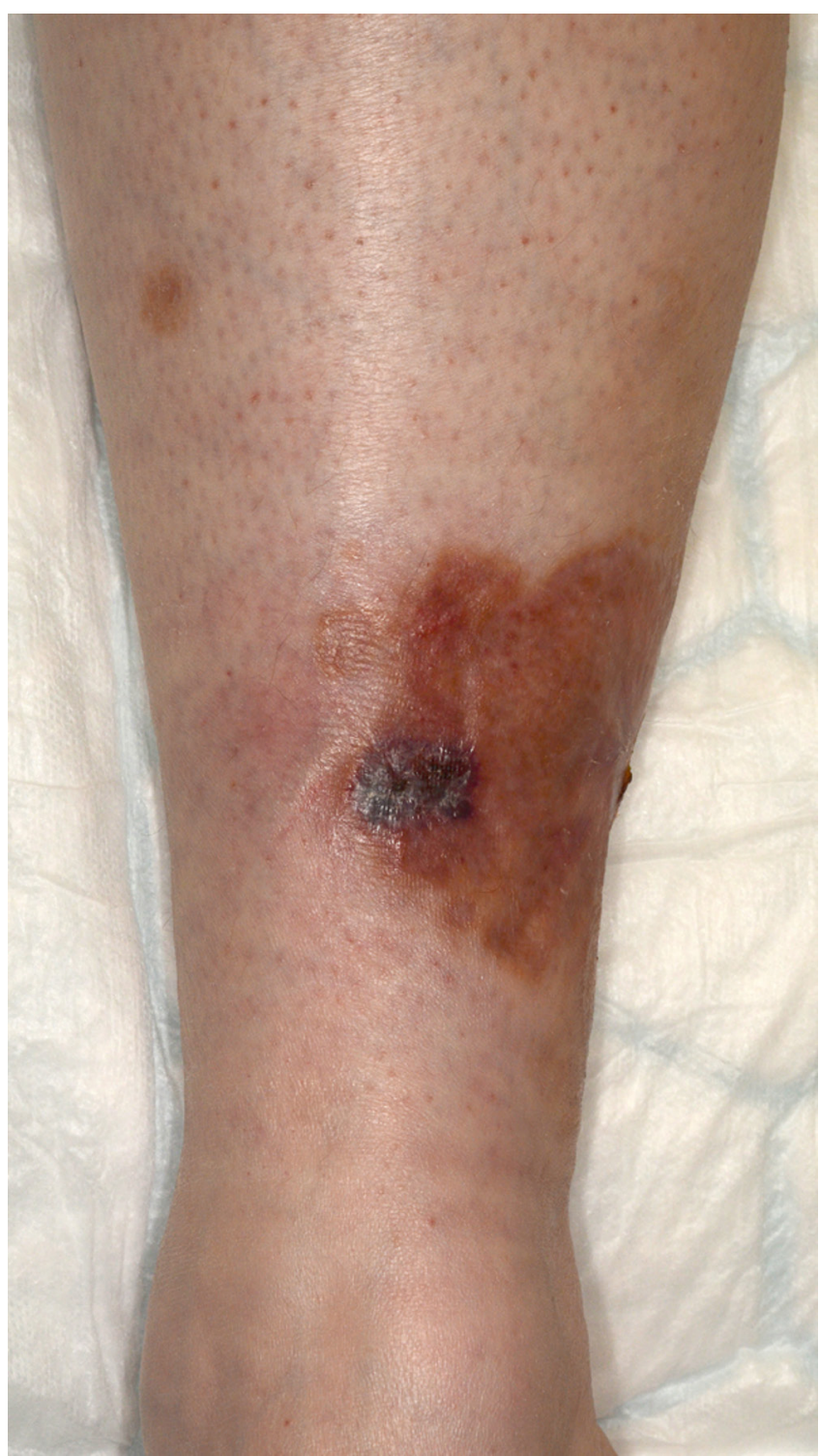

Figure 3 Right shin ulcer healed with previous surrounding ulceration showing epithelisation.

papilloedema, exophthalmos or tenonitis) are detected instead of the original prototypic description of interstitial keratitis. Atypical CS also includes vestibulo-auditory symptoms, which are not typical of Meniere's-like presentation and vestibulo-auditory involvement presenting more than 2 years apart from eye disease.

Due to rare occurrence, there are no international diagnostic criteria; Haynes' proposition of typical and atypical CS is commonly used.

It is recognised as an autoimmune vasculitis, although specific pathogenesis is not fully understood. ${ }^{5}$ Autoantibodies targeting against antigens in the cornea, inner ear and endothelium have been noted, but data are limited on their sensitivity and specificity for clinical diagnosis. ${ }^{6-8}$ It is classified as a variable vessel vasculitis according to the 2012 International Chapel Hill Consensus Conference Nomenclature of Vasculitides, and it is not typically associated with ANCA, which is generally associated with small vessel vasculitis. ${ }^{9}$ The vestibulo-auditory and ocular involvement also often manifests independently of each other, sometimes up to years apart. Therefore, diagnosis can be a challenge and may

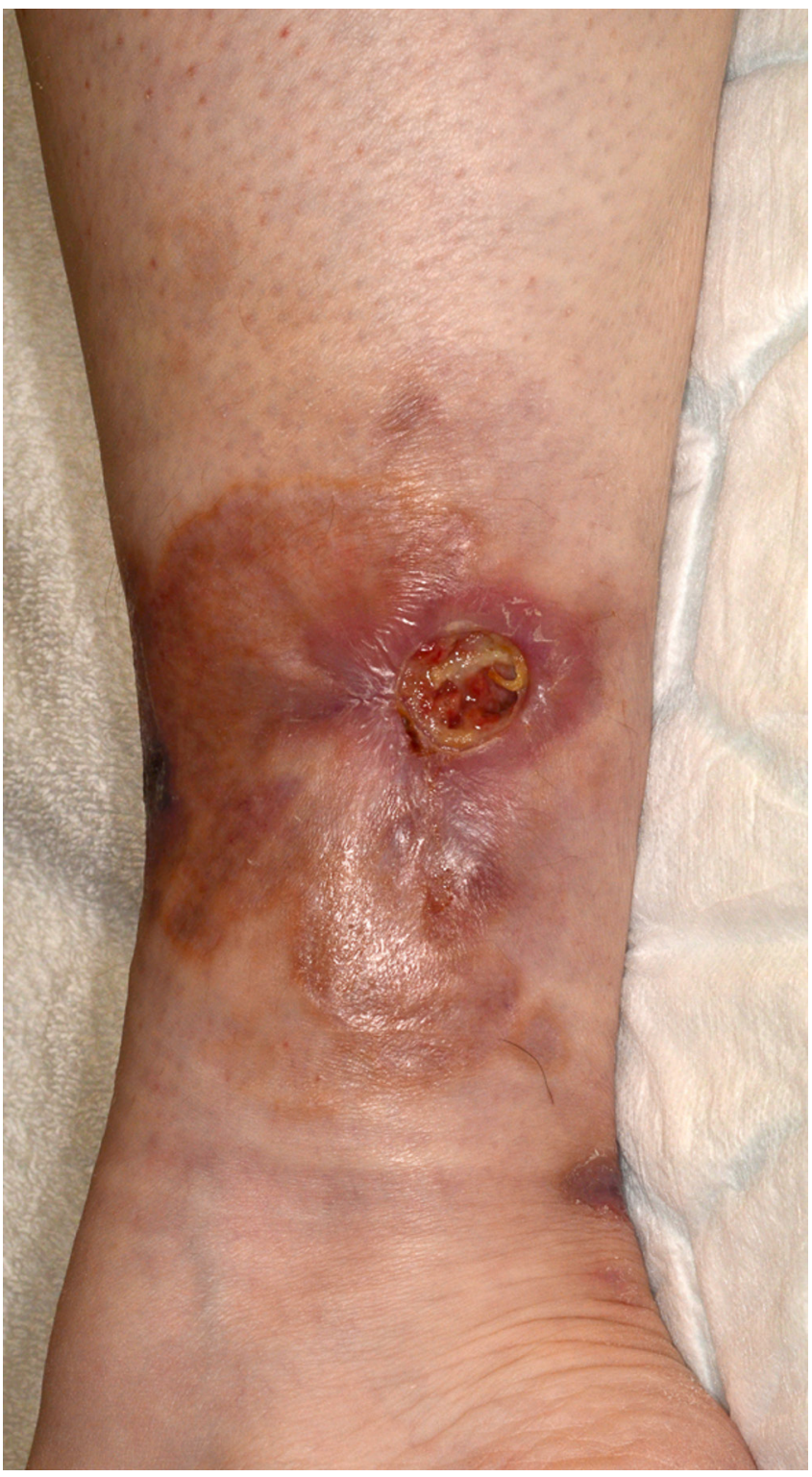

Figure 4 Reduction in size of the right calf ulcer.

be missed due to the wide time gap between manifestations. For these reasons, early diagnosis of CS may be difficult when we rely on case definitions alone. Delayed intervention can result in significant morbidity, from irreversible hearing loss, debilitating vertigo and visual loss. As demonstrated by our patient, she initially presented with acute vestibulo-auditory dysfunction and subtle ophthalmological features. However, with strong suspicion, we initiated high-dose steroids early and this preserved her auditory functions. She further developed classical acute scleritis, which affirmed the diagnosis.

\section{Pyoderma gangrenosum}

PG is a neutrophilic dermatosis of uncertain aetiology. Neutrophilic dermatoses are recently classified as autoinflammatory disorders, usually due to genetic mutation causing dysfunction of the innate immunity. ${ }^{10}$ Classic PG is described as acute spontaneous painful ulcerations with violaceous undermining the borders over the shins. Other variants of pustular PG, pyostomatitis vegetans, bullous PG, vegetative PG and peristomal PG are recognised. The histology in PG is non-specific, but a 


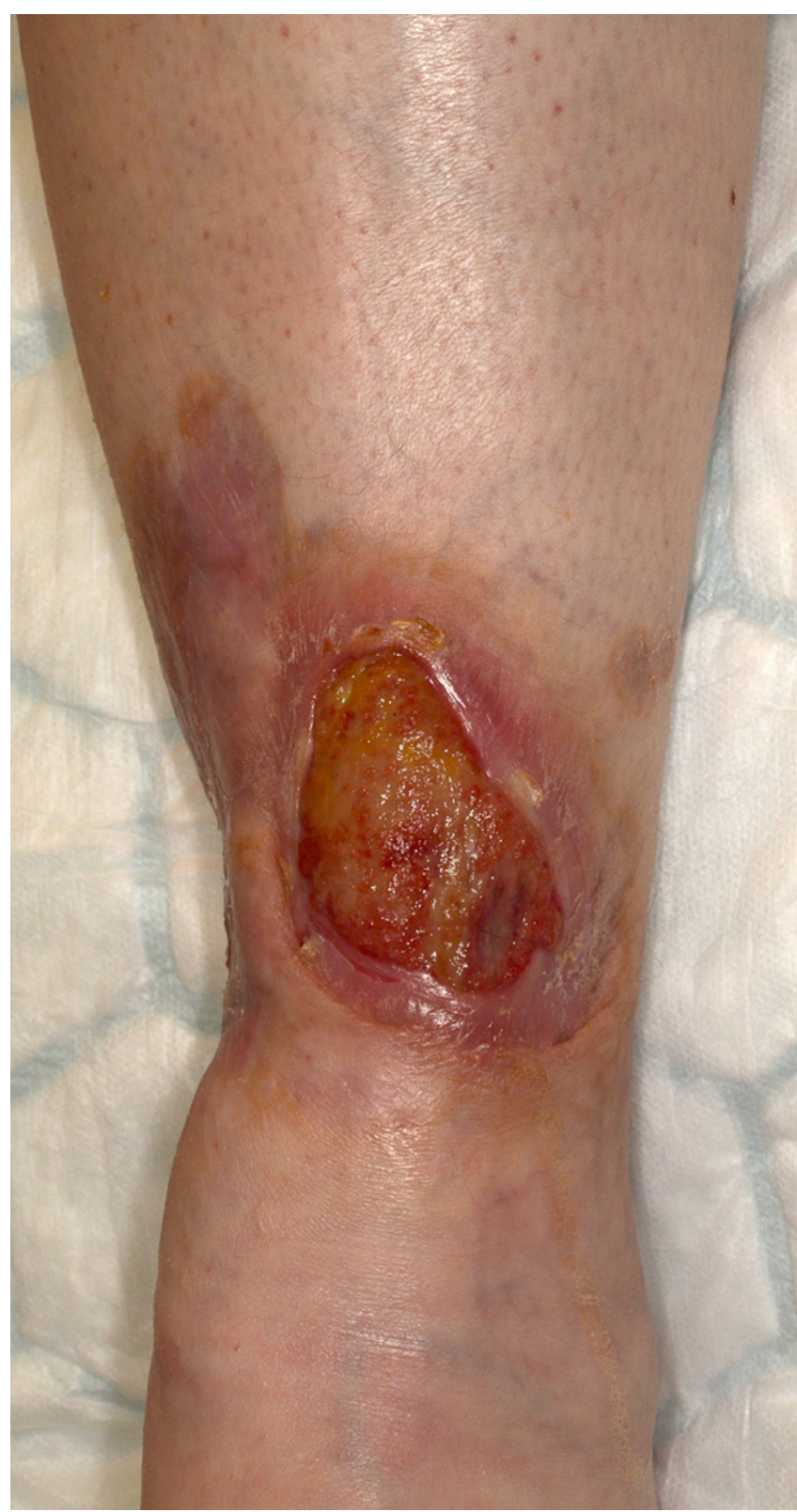

Figure 5 Reduction in size of the left shin ulcer and healthy granulation at the base of the ulcer with epithelising borders.

biopsy helps to exclude other causes of ulcerations. Classically, a dense neutrophilic infiltration, with or without haemorrhage and necrosis of the epidermis, may be present. Blood vessel wall infiltrates may be noted but usually less prominent than that of a primary vasculitis. ${ }^{11}$ PG is also associated with inflammatory bowel disease or haematological malignancies (monoclonal gammopathy and myeloid leukaemia).

\section{Systemic involvement in CS and association with PG}

Other systemic manifestations are also noted in 30\%-50\% of patients with Cogan's. ${ }^{5}$ They include involvement of the cardiovascular system (with large vessel vasculitis, that is, aortitis and aortic insufficiency), neurological system (headaches, lymphocytic meningitis, strokes, transient ischaemic attacks and peripheral neuropathy) and gastrointestinal system (diarrhoea, bleeding and abdominal pain). ${ }^{13}{ }^{14}$ Mucocutaneous manifestations have been described in 12 of 32 patients in a case series by Grasland et al. ${ }^{14}$ These include skin rashes, photosensitivity,

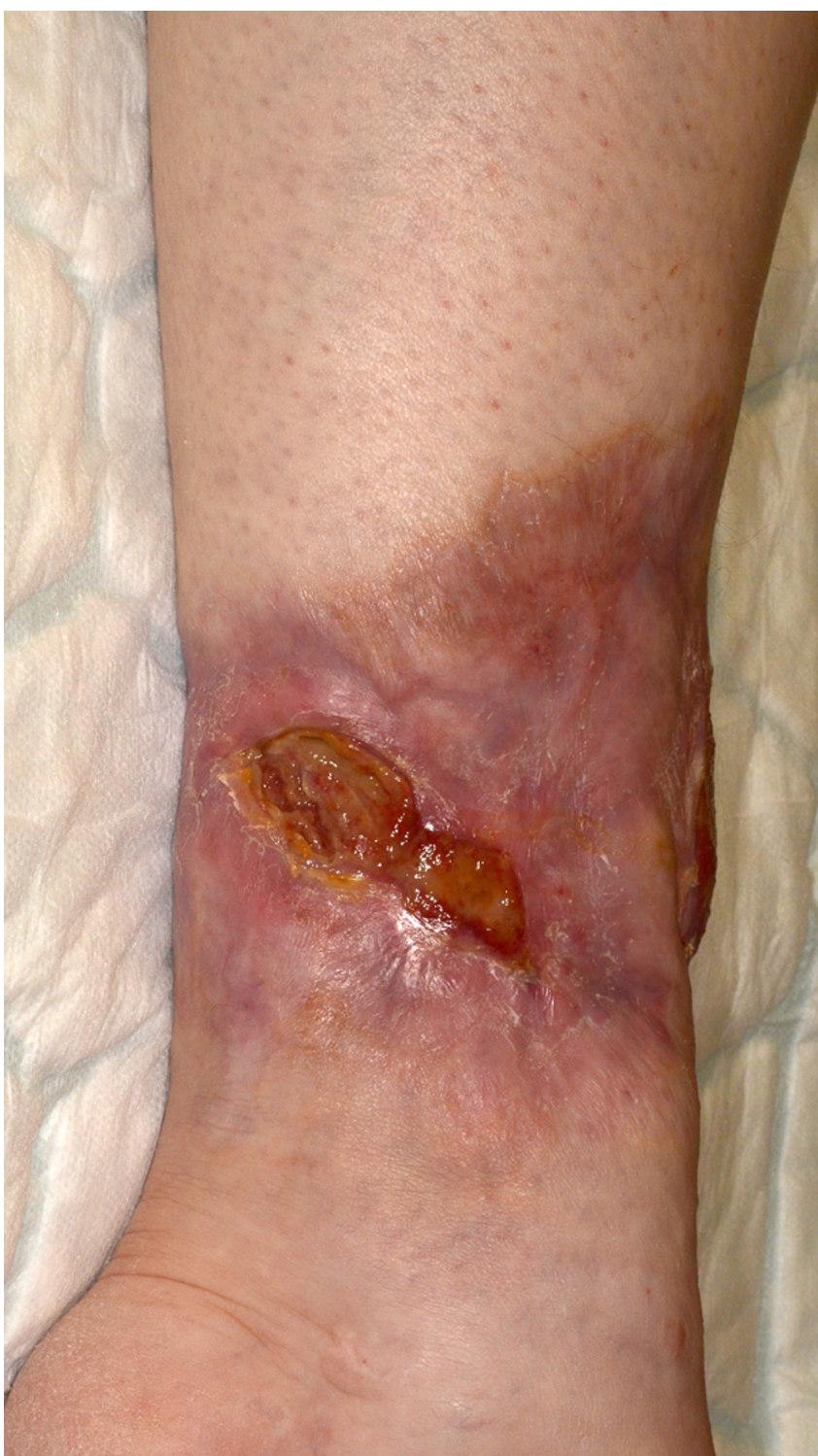

Figure 6 Reduction of the left calf ulcer with surrounding epithelisation of the previous ulceration.

vitiligo, oral ulceration and chondritis. Evidence of vasculitis was present in two skin biopsies. Among paediatric CS, Pagnini et $a l^{15}$ noted 3 of 23 patients with cutaneous manifestations.

More specifically, PG has only been reported in two cases of CS. Boulinguez et $a l^{16}$ reported a 57 -year-old white woman whose uveitis due to CS improved while receiving minocycline for PG in 1998. More recently in 2017, Solano-López et al ${ }^{17}$ reported of a white Spanish woman receiving methotrexate, prednisolone and tocilizumab infusion for CS who developed ulcerative PG. Her ulcers responded to a step-up dose of prednisolone $1 \mathrm{mg} / \mathrm{kg} / \mathrm{day}$. Currently, our patient is a third case report of aggressive PG superimposed by concurrent development of CS despite ongoing escalation of immunosuppression.

\section{Treatment of CS and PG}

There are no guidelines for the treatment of CS or PG due to lack of randomised controlled trials and their uncommon occurrence. Treatment is largely based on observational reports and case series extrapolated from the management of other vasculitides. Corticosteroids are generally first line in CS. Most reports 
use high doses of systemic steroids, such as prednisolone at $1 \mathrm{mg} /$ $\mathrm{kg} /$ day for acute vestibular dysfunction. ${ }^{18}{ }^{19}$ Immunosuppression with oral cyclophosphamide $(1-2 \mathrm{mg} / \mathrm{kg} /$ day $)$, methotrexate, ciclosporin $1-2 \mathrm{mg} / \mathrm{kg} /$ day and azathioprine has been used. ${ }^{13}$ With ocular inflammation, topical steroids are often added. Most recently, a systematic review of 141 adult and paediatric patients demonstrates a general consensus to avoid long-term steroid monotherapy-addition of immunosuppression as steroid sparer is preferred. ${ }^{20}$

In PG, topical steroids and calcineurin inhibitors are frequently used. Severe cases require systemic steroids, with oral ciclosporin for immunosuppression. Dapsone, azathioprine, oral cyclophosphamide, methotrexate, mycophenolate mofetil and IVIG have been used as second-line agents. ${ }^{11}$

So far, there is no evidence of pathophysiological association between both conditions, but anecdotal case reports and case series have suggested some similarity in their management. Our patient manifests both aggressive CS and PG concurrently, with poor response to high-dose steroids with methotrexate, ciclosporin and IVIG. Noting the efficacy of pulsed cyclophosphamide in severe AAV, we used pulsed cyclophosphamide for her vasculitis and are pleased to report that the patient achieved control of activity of both diseases. Cogan's vasculitis is not associated with ANCA, but more robust evidence in the management of such aggressive variable vasculitis is certainly desired.

\section{Patient's perspective}

"I feel the doctors from Rheumatology have achieved a great deal concerning my conditions. At one point, I thought there was nothing anyone can do. With the treatment I have received I do feel more hope with the improvement."

\section{Learning points}

- Cogan's syndrome (CS) is very uncommon compared with strokes and Meniere's disease in acute vestibulo-auditory dysfunction.

- However, it is a consideration when there is a simultaneous or previous event of idiopathic inflammatory eye disease.

- A huge time lapse, sometimes more than 2 years apart, may be present between the involvement of the ocular and auditory system in CS.

- Astute clinical judgement is necessary to pick CS to allow early institution of treatment to prevent irreversible vestibuloauditory damage.

- Biopsies in PG are often non-specific, but in the appropriate clinical context and stereotypical histology the diagnosis can be clinched.

- Repeat skin biopsy may be necessary due to its elusive nature and variable historogical sampling.
Contributors SD and RM managed the patient. SD proposed to write the case as a learning point. EC retrieved the patient's clinical data, drafted the manuscript and arranged the final edition following advice on suggested changes by SD and RM. EC is the corresponding doctor.

Funding The authors have not declared a specific grant for this research from any funding agency in the public, commercial or not-for-profit sectors.

Competing interests None declared.

Patient consent Obtained.

Provenance and peer review Not commissioned; externally peer reviewed.

(c) BMJ Publishing Group Ltd (unless otherwise stated in the text of the article) 2018. All rights reserved. No commercial use is permitted unless otherwise expressly granted.

\section{REFERENCES}

1 Ntatsaki E, Carruthers D, Chakravarty K, et al. BSR and BHPR guideline for the management of adults with ANCA-associated vasculitis. Rheumatology 2014;53:2306-9.

2 Cogan DG. Syndrome of non-syphilitic interstitial keratitis and vestibuloauditory symptoms. Arch Ophthal 1945;33:144-9.

3 Cogan DG. Non syphilitic interstitial keratitis with vestibuloauditory symptoms: Report of four additional cases. Arch Ophthalmol 1949;42:42-9.

4 Haynes BF, Kaiser-Kupfer MI, Mason P, et al. Cogan syndrome: studies in thirteen patients, long-term follow-up, and a review of the literature. Medicine 1980;59:426

5 Greco A, Gallo A, Fusconi M, et al. Cogan's syndrome: an autoimmune inner ear disease. Autoimmun Rev 2013;12:396-400.

6 Arnold W, Gebbers JO. [Serum antibodies against corneal and internal ear tissues in Cogan's syndrome]. Laryngol Rhinol Otol 1984;63:428-33.

7 Arnold W, Pfaltz R, Altermatt HJ. Evidence of serum antibodies against inner ear tissues in the blood of patients with certain sensorineural hearing disorders. Acta Otolaryngol 1985;99:437-44.

8 Helmchen C, Arbusow V, Jäger L, et al. Cogan's syndrome: clinical significance of antibodies against the inner ear and cornea. Acta Otolaryngol 1999;119:528-37.

9 Jennette JC, Falk RJ, Bacon PA, et al. 2012 revised International Chapel Hill Consensus Conference Nomenclature of Vasculitides. Arthritis Rheum 2013;65:1-11.

10 Ahronowitz I, Harp J, Shinkai K. Etiology and management of pyoderma gangrenosum: a comprehensive review. Am J Clin Dermatol 2012;13:191-211.

11 Marzano AV, Ishak RS, Saibeni S, et al. Autoinflammatory skin disorders in inflammatory bowel diseases, pyoderma gangrenosum and Sweet's syndrome: a comprehensive review and disease classification criteria. Clin Rev Allergy Immunol 2013;45:202-10.

12 Cheson BD, Bluming AZ, Alroy J. Cogan's syndrome: a systemic vasculitis. Am J Med 1976;60:549-55

13 Kessel A, Vadasz Z, Toubi E. Cogan syndrome--pathogenesis, clinical variants and treatment approaches. Autoimmun Rev 2014;13:351-4.

14 Grasland A, Pouchot J, Hachulla E, et al. Vinceneux P for the Study group for Cogan's syndrome. Typical and atypical Cogan's syndrome: 32 cases and review of the literature. Rheumatology 2004;43:1007-15.

15 Pagnini I, Zannin ME, Vittadello F, et al. Clinical features and outcome of Cogan syndrome. J Pediatr 2012;160:303-7.

16 Boulinguez $S$, Bernard P, Bedane $C$, et al. Pyoderma gangrenosum complicating Cogan's syndrome. Clin Exp Dermatol 1998;23:286-9.

17 Solano-López G, Montes-Torresa A, Tomero E, et al. Atypical Ulcerated Lesions in a Patient With Cogan Actas Dermo-Sifiliograficas. 2017;108:369-71.

18 Chynn EW, Jakobiec FA. Cogan's syndrome: ophthalmic, audiovestibular, and systemic manifestations and therapy. Int Ophthalmol Clin 1996;36:61-72.

19 Vasileiadis I, Stratoudaki R, Karakostas E. Complete restoration of auditory impairment in a pediatric case of Cogan's syndrome: report of a rare case with longterm follow-up and literature review. Int J Pediatr Otorhinolaryngol 2012;76:601-5.

20 Mora P, Calzetti G, Ghirardini S, et al. Cogan's syndrome: State of the art of systemic immunosuppressive treatment in adult and pediatric patients. Autoimmun Rev 2017:16:385-90 


\section{Unusual association of diseases/symptoms}

Copyright 2018 BMJ Publishing Group. All rights reserved. For permission to reuse any of this content visit http://group.bmj.com/group/rights-licensing/permissions.

BMJ Case Report Fellows may re-use this article for personal use and teaching without any further permission.

Become a Fellow of BMJ Case Reports today and you can:

- Submit as many cases as you like

- Enjoy fast sympathetic peer review and rapid publication of accepted articles

- Access all the published articles

- Re-use any of the published material for personal use and teaching without further permission

For information on Institutional Fellowships contact consortiasales@bmjgroup.com

Visit casereports.bmj.com for more articles like this and to become a Fellow 